\title{
Serum Alpha Fetoprotein (AFP) Levels in Normal Infants
}

\author{
JAMES T. WU, ${ }^{(14)}$ LINDA BOOK, AND KAREN SUDAR \\ Departments of Pathology and Pediatrics, University of Utah Medical Center, Salt Lake City, Utah, USA
}

\begin{abstract}
Summary
Serum $\alpha$-fetoprotein (AFP) levels were monitored in 32 normal babies consecutively from 2 to 3 days, 2 wk, and 2 and 4 months after birth. In addition, serum AFP concentration was also measured in 116 random specimens from infants with normal liver enzymes and 10 infants born immaturely. Results were combined to establish normal AFP levels for infants at various ages. Serum AFP disappeared rapidly after birth. We found that it was not until 8 months of age that the normal AFP level in infants approached adult level. The half-lives of AFP degradation were estimated to be 5.5 days between birth and $2 \mathrm{wk}$, 11 days between 2 wk to 2 months, and 33 days between 2 and 4 months of age. In contrast to earlier belief, we felt that some AFP synthesis still exists after birth; however, the rate of synthesis may also decrease with age.
\end{abstract}

\section{Speculation}

Our results favor the view that some $\alpha$-fetoprotein (AFP) synthesis exists after birth. The rate of synthesis is slowly decreased with time. Any factor affecting the rate of synthesis may influence the serum AFP level. The wide range of variation in serum AFP levels in infants of various ages indicates that there must be more than one unknown factor affecting the AFP serum level. The normal range will be narrower if these factors become known. By using our age-dependent normal AFP serum levels, it would be interesting to see whether differential diagnosis of neonatal hepatitis from biliary atresia can be improved and whether infants do have higher and more frequent elevation of serum AFP with hepatic disorders.

In adults, the measurement of serum $\alpha$-fetoprotein (AFP) level is useful in the diagnosis and treatment of hepatoma, teratoblastoma, and a number of liver diseases $(3,10)$. However, because of the lack of reliable normal values for infants, the diagnosis of similar abnormalities in these patients is more difficult. At birth, the average AFP level of cord serum is several thousandfold higher than are adult levels. These levels progressively decline as the infant matures $(7,11,12)$ However, it is not precisely known at what age the serum AFP of infants reaches adult level. In addition, the rate at which the level declines with age, as well as the various normal levels during that period, are also unknown.

Several investigators have attempted to distinguish infants with neonatal hepatitis from those with biliary atresia by measuring their serum AFP level $(2,7,12)$. In this regard, a single normal AFP level was used, although the infants varied by several months of age. It is possible that this differential diagnosis may be significantly improved if more reliable age-discriminated normal AFP levels were available. In our study, we monitored the serum AFP levels of 32 normal babies consecutively from birth to 4 months of age. In addition, AFP levels were determined in 116 random serum specimens from supposedly normal children with normal serum liver enzyme activities to establish acceptable upper normal limits for these older infants and to determine at what age they fall to adult level.

\section{MATERIALS AND METHODS}

Capillary blood specimens were obtained from 32 normal babies consecutively at birth and at 2 wk, 2 months, and 4 months of age. An additional 116 random specimens from infants less than one year old with normal liver enzyme activities were also included. Ten additional serum specimens were obtained from normal premature babies one to two wk of age. Informed written parental consent was obtained for the 32 newborns before participation in the study. Additional 116 serum samples were obtained from healthy infants and children primarily admitted for elective surgery who were undergoing routine preoperative laboratory evaluation. The tested liver enzymes included alkaline phosphatase, lactic dehydrogenase, aspartate transaminase, and gamma glutamyltransferase. Concentration of serum AFP was determined by a double antibody radioimmunoassay with a sensitivity of $2 \mathrm{ng} /$ $\mathrm{ml}$. The assay requires only $50 \mu \mathrm{l}$ of sample. The AFP standard was calibrated against the International Reference Preparation $72 / 227$. Inasmuch as most of the infant serum specimens contained much higher AFP concentrations than adult samples, proper dilution with phosphate-buffered saline containing $1 \%$ albumin is required before the assay. The extent of the dilution is age dependent and should be such that the concentration falls in the middle range of the AFP standard curve. All specimens were stored at $4^{\circ} \mathrm{C}$ and assayed within 1 to $2 \mathrm{wk}$.

\section{RESULTS}

To demonstrate the rate of decline of AFP in individual infants, four levels of serum AFP of 32 normal infants at 2 to 3 days, 2 wk, 2 months, and 4 months after birth were plotted and connected by straight lines. The result is shown in Figure 1. To accommodate the wide range of AFP levels and to facilitate the estimation of half-life of AFP, a semi-logarithmic plot was used. The half-life of AFP degradation (which is the time interval corresponding to a decrease of $\log 2$ or the time at $\log$ (AFP) concentration at day zero/2] can be estimated graphically. In general, the rate of decline was most rapid between birth and 2 wk of age. The half-life of AFP degradation during this period was estimated to be 5.5 days according to first-order kinetics. The rate of decline decreased between 2 and 8 wk of age and further decreased after 2 months of age. The average half-lives of AFP beween 2 wk to 2 months and 2 to 4 months were 11 and 33 days, respectively.

Individual serum AFP levels at any age were found to be highly variable. No correlation between the level of AFP with birth weight, sex, gestational age, type of milk feeding, presence or absence of jaundice, etc., was noticed. For the majority of the infants, the rate of AFP decline was very similar. Consequently, an infant with high levels at birth usually maintained relatively higher levels of AFP throughout the entire period of early infancy. However, deviation from the normal pattern of decline was also 


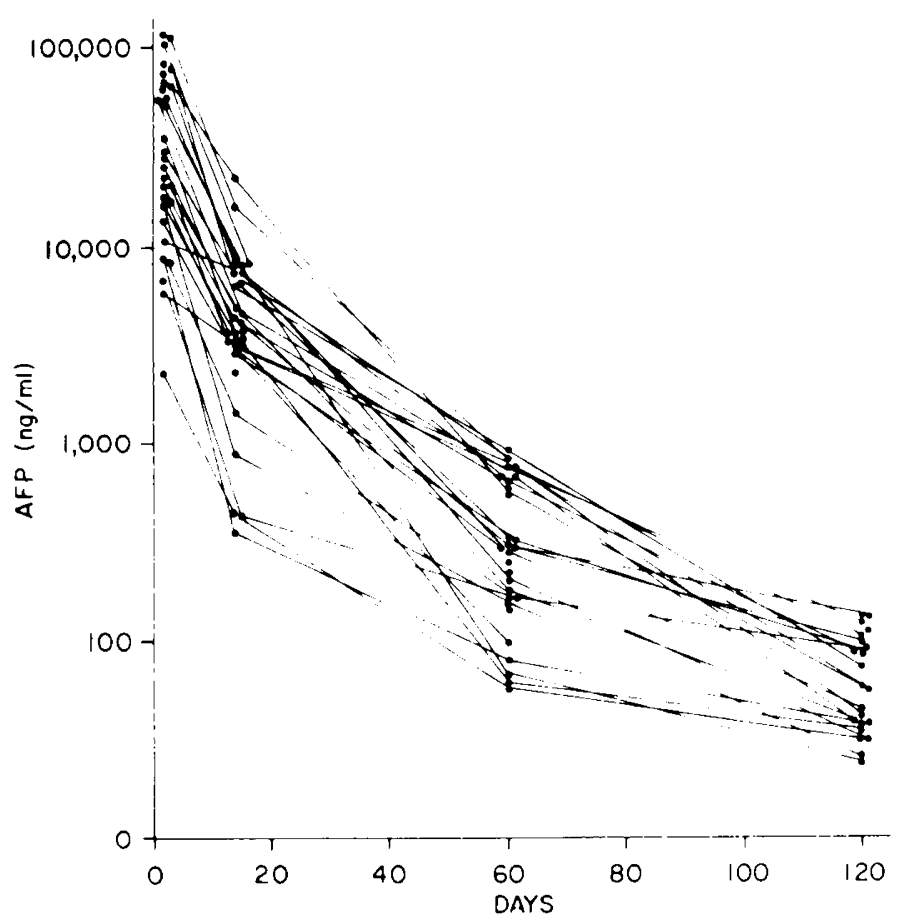

Fig. 1. Serum AFP levels of 32 normal babies measured consecutively at 2 or 3 days, $2 \mathrm{wk}$, and 2 and 4 months after birth.

observed in small number of infants. The reason for this deviation was not clear.

To establish normal AFP levels for infants at various ages and to determine at what age their AFP levels approach adult level, the values used in Figure 1 were replotted in Figure 2. In addition, 116 random specimens without elevated liver enzymes along with the 10 samples obtained from premature infants were also included. Because there was only a limited number of specimens for any defined age, they were divided into several groups, namely, premature, newborn, from birth to two wk, two wk to one month, and monthly thereafter. The need to separate the infants before and after two wk of age is due to the rapid decline during this early period. For any individual infant, it is apparent from Figure 1 that a very large change of AFP occurs over the first 2 wk of life. However, further refined grouping of these results may not be practical because of the large variation of the normal levels at any age of the infants.

The results presented in Figure 2 indicate that after 8 months of age AFP level of infants have reached adult level. The average normal serum AFP level and its standard deviation of each age group are summarized in Table 1 . Because our results show no difference in AFP levels between infants after 8 months of age and adult, only AFP level up to 8 months are listed. We have measured AFP level in 50 normal adult blood bank donors. The average serum AFP level was found to be $4.7 \pm 3 \mathrm{ng} / \mathrm{ml}$. The value of mean plus 2 :tandard deviations was plotted in Figure 2 as upper normal limit.

\section{DISCUSSION}

It has been reported (8) that serum AFP disappears from blood $2 \mathrm{wk}$ after birth. Most likely it was due to the lack of sensitivity of the technique they used that levels below $\mu \mathrm{g} / \mathrm{ml}$ range could not be detected. Masseyeff $e t$ al. (11) first demonstrated the gradual decline of serum AFP postnatally using a sensitive radioimmunoassay technique. Their results indicated that it may be a year or so before the infant's AFP level approached that of adults. However, because of the limited number of random specimens included in their study, no reliable normal levels could be established for infants. In this study, we report AFP levels in 148 serum specimens

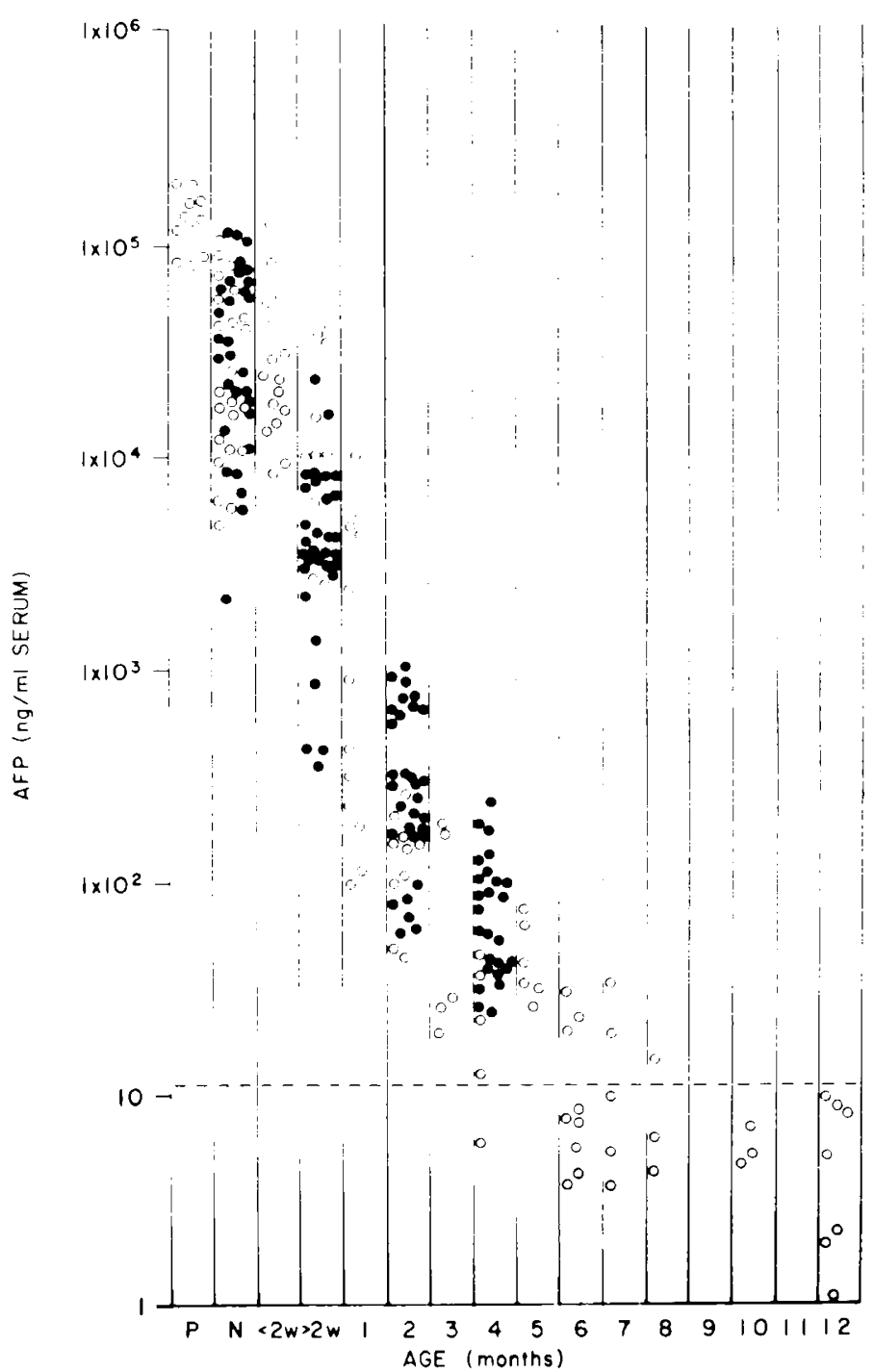

Fig. 2. Serum AFP levels of normal infants and infants with normal liver enzymes. 9 , serum AFP levels of 32 normal babies which were plotted in Figure $1 ;$ O, AFP levels of 116 random specimens. Serum AFP levels from 11 premature infants are also included. Dotted line, normal upper level for adults. $P$, premature: $N$, newborn; $<2 w$, newborn to $2 w \mathrm{k}$ : $>2 w, 2$ wk to one month of age.

Table 1. Average normal serum AFP of infants at various ages

\begin{tabular}{lcc}
\hline \multicolumn{1}{c}{ Age } & No. & Mean \pm S.D. $(\mathrm{ng} / \mathrm{ml})$ \\
\hline Premature & 11 & $134.734 \pm 41.444$ \\
Newborn & 55 & $48.406 \pm 34.718$ \\
Newborn-2 wk & 16 & $33.113 \pm 32.503$ \\
2 wk-1 mo. & 43 & $9.452 \pm 12.610$ \\
1 mo. & 12 & $2.654 \pm 3.080$ \\
2 mos. & 40 & $32.3 \pm 278$ \\
3 mos. & 5 & $88 \pm 87$ \\
4 mos. & 31 & $74 \pm 56$ \\
5 mos. & 6 & $46.5 \pm 19$ \\
6 mos. & 9 & $12.5 \pm 9.8$ \\
7 mos. & 5 & $9.7 \pm 7.1$ \\
8 mos. & 3 & $8.5 \pm 5.5$
\end{tabular}

from infants between birth and one year of age. Our results indicate that AFP levels in infants reach adult levels by 8 months of age. It is interesting to note that higher levels of serum AFP may be seen in pediatric hepatic disorders $(3,9)$ than adults. Moreover, Abelev et al. (1) found that the production of AFP after 
toxic injury to liver cell was more easily elicited in young animals. Therefore, it seems that the normal AFP levels provided by this study should improve the diagnostic value of serum AFP measurements for infants under one year of age.

We also hope that the age-dependent normal AFP levels provided in this study would improve the differential diagnosis of neonatal hepatitis from biliary atresia. Differential diagnosis between extrahepatic biliary atresia and neonatal hepatitis is difficult either on clinical or biochemical ground. Andres et al. (2) proposed that serum AFP levels greater than $35 \mu \mathrm{g} / \mathrm{ml}$ in infants 1 to 4 months of age could be used for the diagnosis of neonatal hepatitis whereas values below $10 \mu \mathrm{g} / \mathrm{ml}$ in infants under 4 months of age be used for the diagnosis of biliary atresia. Johnston et al. (7), on the other hand, indicated that AFP concentration greater than 10 $\mu \mathrm{g} / \mathrm{ml}$ favored the diagnosis of neonatal hepatitis especially in the first 10 wk of life. It is apparent from the values listed in Table 1 that using either 35 or $10 \mu \mathrm{g}$ AFP per $\mathrm{ml}$ for the diagnosis of neonatal hepatitis in those age ranges would undoubtedly yield many false negatives because the normal AFP levels in both groups of patients are much lower than the cutoff levels used. On the other hand, using $10 \mu \mathrm{g} / \mathrm{ml}$ to differentiate neonatal hepatitis from biliary atresia in the first 10 wk of life (7) would probably yield both false positives in infants less than 2 wk of age and false negatives in infants between 2 and 10 wk.

The half-life of 5.5 days estimated by us for AFP degradation between birth and 2 wk of age is in close agreement with reports from other investigators $(5,6)$. However, we found that the degradation rate decreased considerably after that initial period. It was pointed out by several investigators that there was essentially no synthesis of AFP immediately after birth because of the rapid decline observed in serum AFP postnatally $(5,6)$. However, our results shown in Figure 1 indicate that a small amount of AFP is continuously synthesized. By following the serum AFP level in each individual infant, the rate of decline of serum AFP does not follow a first-order kinetics for the entire 4 months after birth. If the rate of decline is only controlled by AFP turnover rate, a semilogarithmic plot would have yielded a straight line. Therefore, it is most likely that some residual synthetic activity exist postnatally. In rat, Colguhoun et al. (4) also observed a biphasic pattern of AFP disappearance from the circulation of the neonatal rat, indicating possible continued AFP synthesis after birth. In our case, the amount of AFP synthesized may not be significant compared to the amount of AFP in the serum of infants during the first 2 wk of life. The rate of decline is therefore equivalent to AFP turnover rate. However, within 2 wk after birth, the serum AFP level is much lower, and the rate of AFP decline is no longer controlled by AFP turnover alone. Instead, the small amount of AFP synthesized begin to affect the degradation pattern. This effect becomes even more apparent after 2 months of life when the residual serum AFP level approaches adult level. This explains the result of a curved line instead of a straight one as we show in Figure 1. It should be noted that even the rate of synthesis may decline slowly during the first year of life. After 8 months of age, only trace AFP synthesis exist.

Similar to what was observed by Masseyeff et al. (11), we also notice a wide range of variation of normal AFP levels in infants at all ages. This wide range of variation results not only in considerable overlap between normal levels of different age groups, but also in large standard deviations as shown in Table 1. We hope that additional unknown factors affecting AFP level will be discovered in the future, which should help to narrow the agedependent normal AFP values and further improve the diagnostic value of measuring AFP in serum.

By monitoring the serum AFP levels of 32 normal babies consecutively from birth to 4 months of age and by measuring AFP levels in additional 116 random serum specimens from supposedly normal children with normal serum liver enzyme activities, we have established normal serum AFP levels for infants from birth to 8 months of age. The serum AFP levels are age dependent; they gradually decline as infant matures. It was not until 8 months of age that the serum AFP levels in infants approach adult level. The rate of decline of serum AFP also change with age. The half-lives were estimated to be 5.5 days between birth and 2 wk of age, 11 days between 2 wk and two months, and 33 days between 2 and 4 months. The change of the rate of AFP degradation indicate that a considerable amount of AFP synthesis exists after birth.

\section{REFERENCES AND NOTES}

I. Abelev, G. I.: Alpha-fetoprotein in ontogenesis and its association with malignant tumors. Adv. Cancer Res., 14: 295 (1971).

2. Andres, J. M., Lilly,. J. R., Altman, R. P., Walker, W. A., and Alpert, E.: Alphafetoprotein in neonatal hepatobiliary disease. J. Pediatr., 91: 217 (1977).

3. Bloomer, J. R., Waldmann, T. A., McIntire, K. R., and Klatskin, G.: $\alpha$-Fetoprotein in nonneoplastic hepatic disorders. J. Am. Med. Assoc., 233: 38 (1975).

4. Colguhoun, B., Gold, P., Brazell, W., Oakes, D. D., and Shuster, J.: Metabolism of rat $\alpha_{1}$-fetoprotein. Cancer Res., 34: 2296 (1974).

5. Gitlin, D., and Boesman, M.: Serum $\alpha$-fetoprotein, albumin, and $\gamma$-globulin in the human conceptus. J. Clin. Invest., 45: 1826 (1966).

6. Hyvarinen, M., Zeltzer, P., Oh, W., and Stiehm, E. R.: Influence of gestational age on serum levels of alpha-I-fetoprotein $\mathrm{IgG}_{\mathrm{g}}$ globulin, and albumin in newborn infants. J. Pediatr., 82: 430 (1973).

7. Johnston, D. T., Mowat, A. P., Orr, H., and Kohn, J.: Serum alpha-fetoprotein levels in extra hepatic biliary atresia idiopathic neonatal hepatitis and alpha-1antitrypsin deficiency. Acta Pediatr. Scand., 65: 623 (1976).

8. Karlsson, B. W., Bergstrand, C. G., Ekelund. H., and Lindberg. T.: Postnata] changes of alpha-fetoprotein. Albumin and total protein in human serum. Acta Pediatr. Scand., 61: 133 (1972).

9. Ken, M. C., Purves, L. R., and Bersohn, I.: Serum alpha-fetoprotein levels in acute viral hepatitis. Gut, 14: 939 (1973).

10. Mann, J. R., Lakin, G. E., Leonard, J. C., Rawlinson, H. A., Richardson, S. G. N., Corkery, J. J., Cameron. A. H., and Shah, K. J.: Clinical applications of serum carcinoembryonic antigen and alpha-fetoprotein levels in children with solid tumours. Arch. Dis. Child., 53: 366 (1978).

11. Masseyeff, R., Gilli, J., Krebs, B., Calluaud, A., and Bonet, C.: Evolution of $\alpha-$ fetoprotein serum levels throughout life in humans and rats, and during pregnancy in the rat. Ann. N. Y. Acad. Sci., 259: 17 (1975)

12. Zeltzer, P. M., Neerhout, R. C., Fonkalsrud, E. W., and Stiehm, R.: Differentiation between neonatal hepatitis and biliary atresia by measuring serum alphafetoprotein. Lancet. $l: 7854$ (1974).

13. The authors would like to thank Russell Haymond and Dr. Joseph Knight for providing 116 random specimens from Primary Children's Hospital. We also want to thank Drs. J. Knight and G. Chan for helpful discussions.

14. Requests for reprints should be addressed to: James $T$. Wu, Department of Pathology, University of Utah Medical Center, Salt Lake City, UT 84132 (USA).

15. This research was supported by the development fund from the Department of Pathology, University of Utah Medical Center.

16. Received for publication April 1, 1980.

17. Accepted for publication June 6, 1980. 